\title{
Comportamento da artéria caudal bovina como enxerto biológico vascular alternativo: estudo experimental em cães. (Nota prévia)
}

\author{
Mauro Barbosa ARRUDA FILHO*, Mauro Barbosa ARRUDA*
}

RBCCV $44205-324$

\begin{abstract}
Arruda Filho M B \& Arruda M B - Comportamento da artéria caudal bovina como enxerto biológico vascular alternativo: estudo experimental em cães. (Nota prévia). Rev Bras Cir Cardiovasc 1996; 11 (4): 299 306.

RESUMO: Foram interpostos 20 segmentos de artéria caudal bovina (ACB), tratados com glutaraldeído e formaldeido, nas artérias femorais de 10 cães. O período de seguimento pós-operatório variou de 180 a 297 dias. Foram avaliados: função motora, pulsos (à palpação e ao ultra-sônar), variações das pressões arteriais sistólica, diastólica e média e fluxo através do enxerto, assim como consistência dilatação e coloraçāo quando da retirada. Não houve óbitos, alteraçōes da marcha ou processos inflamatórios nas feridas. Houve trombose total $(15 \%)$, trombose mural focal $(30 \%)$, reação linfoplasmocitária focal da adventícia (100\%), calcificação unicamente das miocélulas $(5 \%)$, reendotelizaçāo total $(60 \%)$ e parcial $(30 \%)$. Por apresentar adequaçāo com diâmetro de pequenas artérias, ter comprimento suficiente para substituir ou atingir diversos segmentos de artérias distais, apresentar rápida endotelização, bem como ausência de rejeição, é demonstrada a receptividade ao uso da ACB como enxerto vascular alternativo.
\end{abstract}

DESCRITORES: Artéria femoral, cirurgia. Bioprótese, métodos. Materiais biocompatíveis.

\section{INTRODUÇÃO}

Uma das controvérsias em operações de pequenas artérias e revascularização do miocardio é a escolha de um enxerto substituto, quando os enxertos autológos não estão disponíveis.

A veia safena magna figura entre os enxertos autólogos mais utilizados, com seguimento evolutivo acima de quinze anos (1-3), embora o desenvolvimento da doença arterioesclerótica e a aceleração da hiperplasia intimal constituam problemas freqüentes (3-6).
A artéria torácica interna tornou-se 0 enxerto ideal nas operações de revascularização do miocárdio, mas, pela sua localização, tem pouca aplicabilidade e disponibilidade outras, se não nestes procedimentos (7-9).

As artérias gastroepiplóica direita, radial, epigástrica inferior e esplênica vêm sendo utilizadas como enxertos alternativos; entretanto, seu seguimento clínico, por ser recente, ainda merece melhor avaliação (10-14).

As próteses sintéticas produzidas em PTFE e

Trabalho realizado no Serviço de Cirurgia Cardiovascular e Torácica - Real Hospital Português em Pernambuco. Núcleo de Cirurgia Experimental do HC.UFPE e LIKA UFPE. Recife, PE, Brasil.

Apresentado ao $23^{\circ}$ Congresso Nacional de Cirurgia Cardiaca. Recife, PE, 20 a 23 de março, 1996.

* Do Serviço de Cirurgia Cardiovascular e Torácia do Real Hospital Português em Pernambuco.

Endereço para correspondẻncia: Mauro Barbosa Arruda Filho. Rua Gervásio Fioravante 87/202. Graças. Recife, PE, Brasil. CEP: 52011-030. Tel: (081) 423-5496. 
Arruda Filho M B \& Arruda M B - Comportamento da artéria caudal bovina como enxerto biológico vascular alternativo: estudo experimental em cāes. (Nota prévia). Rev Bras Cir Cardiovasc 1996; 11 (4):299-306 .

Dacron têm demonstrado resultados insatisfatórios nas operações de revascularização miocardica, sendo utilizadas com freqüência nas operações de bypass artério-arterial e na construção de shunts arteriovenosos, com resultados variáveis (15-17).

Os heteroenxertos arteriais bovinos foram utilizados a partir de Rozemberg, em 1955. Foram empregados como enxertos alternativos em desvios subclávio-braquial, na feitura de fístulas para hemodiálise, no reparo de defeitos traqueais. Recentemente, estes enxertos surgiram no mercado para revascularização do miocárdio e em operações de pequenas artérias. Entretanto, mostram variação de patência de estudo para estudo, seja pela inadequação dos diâmetros das artérias e serem revascularizadas, seja pela diferença de textura das paredes e pelo método de preservação e/ou conservação empregados (18-21).

A presente investigação é um estudo experimental do comportamento da artéria caudal bovina como substituto de segmentos de artérias femorais em cães. Seu objetivo é a busca de um conduto biológico alternativo, que tenha uma adequação com o diâmetro das artérias a serem revascularizadas, tenha comprimento suficiente para atingir ou substituir diversos seguimentos de artérias bem distais, que seja de fácil obtenção, que possua estrutura histológica semelhante aos vasos nativos, que tenha capacidade de rápida endotelização e que seja economicamente viável.

\section{MATERIAL E MÉTODOS}

\section{Preparação do Enxerto de Artéria Caudal Bovina (ACB)}

Os espécimes de, artéria caudal bovina foram colhidos imediatamente após o abate, em matadouro público autorizado pelo Ministério da Saúde, sendo os animais machos, com idade variando de 30 a 60 meses e devidamente inspecionados pela legislação vigente.

A dissecção foi cuidadosamente realizada, para evitar danos às $A C B$, que possuem comprimento médio de $70 \mathrm{~cm}$. Logo após a dissecção, os segmentos de ACB foram acondicionados em recipientes com solução hipertônica da $\mathrm{NaCl}$ tamponada com MgSO4 8 mos a Ph 7,4, com tampão fosfato a uma temperatura de $4^{\circ} \mathrm{C}$, por um período de quatro horas, seguindo-se já em ambiente estéril e sob condições de assepsia e anti-sepsia; a primeira limpeza retirando-se o tecido adiposo junto à adventícia e a ligadura de seus ramos com pontos isolados de Polipropileno 6-0. As fases em seqüencia até a preparação final estão representadas no Quadro 1.

QUADRO 1

PREPARAÇĀO DO ENXERTO DE ARTÉRIA CAUDAL BOVINA

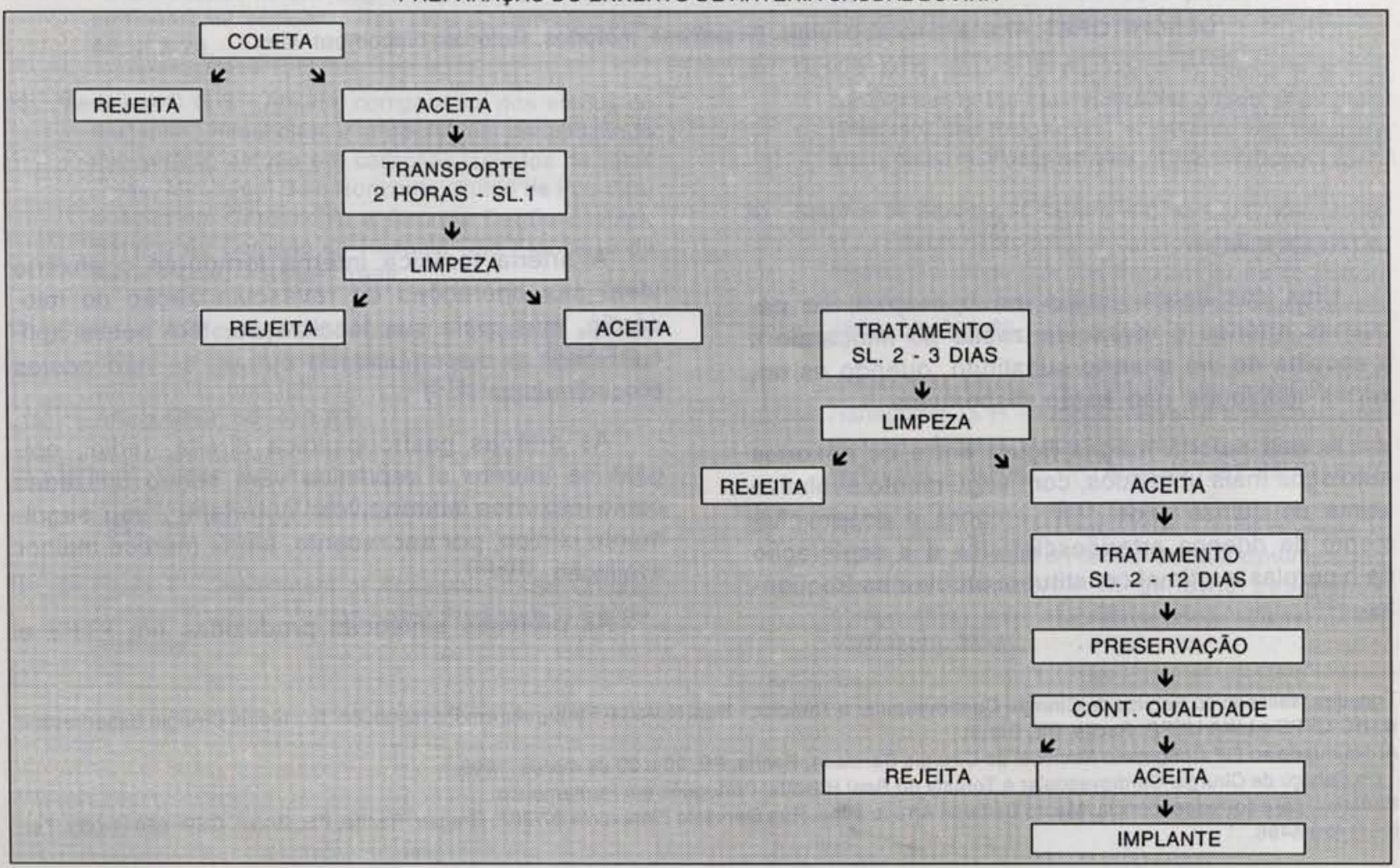


Arruda Filho M B \& Arruda M B - Comportamento da artéria caudal bovina como enxerto biológico vascular alternativo: estudo experimental em cāes. (Nota prévia). Rev Bras Cir Cardiovasc 1996;11 (4):299-306 .

\section{Protocolo Experimental}

Foram interpostos segmentos medindo cerca de $3 \mathrm{~cm}$, de enxertos de $A C B$, nas artérias femorais direita e esquerda de 10 cães mestiços, com idade variando de 28 a 32 meses, peso médio de $11,26 \mathrm{~kg}$, no Núcleo de Cirurgia Experimental do Hospital das Clínicas da UFPE. (Tabela 1).

Os cães foram submetidos a exame clínico, vacinação, quarentena e tratamento antiparasitário. Não apresentavam alteração da marcha ou de movimentação da cauda, tiveram os pulsos femorais aferidos pela palpação, análise com estetoscópio ultra-sônico EU 700, pressão arterial sistólica e diastólica aferidas nos membros inferiores utilizando-se tensiomêtro marca Labtron. Estas medidas foram utilizadas como parâmetro para acompanhamento e observação pós-operatória (Tabela 1).

\section{Técnica de Implante}

Os animais de experimentação foram preparados utilizando-se como agente pré-anestésico o Midazolan na dose $0,15 \mathrm{mg} / \mathrm{kg} / \mathrm{pes}$, por via subcutânea, trinta minutos antes da realização do procedimento. As cirurgias foram realizadas em ambiente asséptico, sob anestesia geral intravenosa, utilizando-se cloridrato de ketamina na dose de $0,2 \mathrm{mg} / \mathrm{kg} /$ peso e o brometo de pancurônio na dose de 0,08 $\mathrm{mg} / \mathrm{kg} /$ peso. Foi utilizado ventilador Takaoka modelo 6000 , com volume corrente de $15 \mathrm{ml} / \mathrm{kg} /$ peso.

Em condições assépticas, a incisão no terço médio da coxa foi realizada, sendo a artéria femoral dissecada e isolada. Os ramos-arteriais ao nivel e abaixo do segmento escolhido para interposição do enxerto foram ligados com fios de algodão $4-0$ sec-

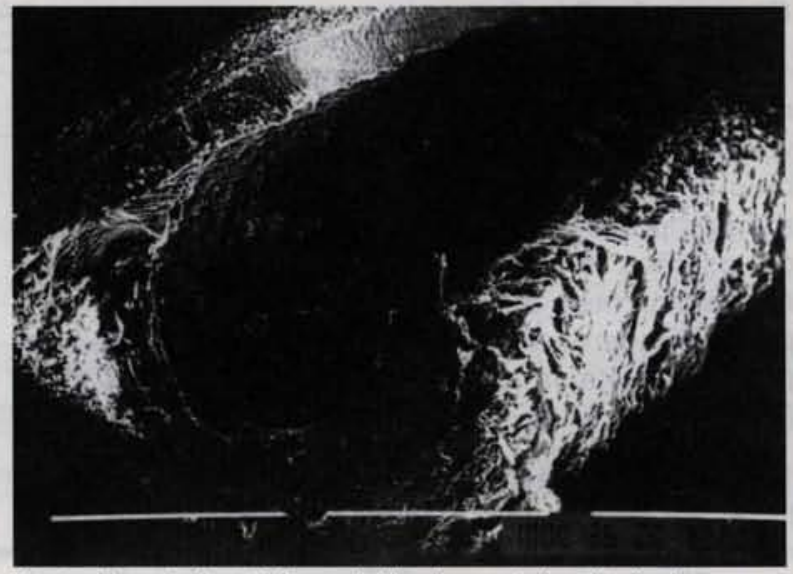

Fig. 1 - Enxerto da artéria caudal bovina; corte longitudinal

cionados, para evitar fluxo colateral para o seguimento inferior ao enxerto. Antes do clampeamento arterial, $0,01 \mathrm{mg} / \mathrm{kg} / \mathrm{peso}$ de heparina foi administrado por via intravenosa. A artéria femoral foi, então, pinçada proximal e distalmente, um segmento medindo cerca de $2 \mathrm{~cm}$ foi excisado e realizada a interposição término-terminal de um segmento de ACB medindo cerca de $3 \mathrm{~cm}$, com sutura contínua com fio de monofilamento de Polipropileno 7-0. Após o desclampeamento, foi revisada a hemostasia e a ferida fechada por planos anatômicos, sendo realizada sutura intradérmica com fio de Mononylon 5-0.

Em seguida, obedecendo à mesma técnica descrita, foi realizada a interposição na artéria femoral contra-lateral. Ao seu término, a reversão anestésica foi feita com Neostigmine $0,06 \mathrm{mg} / \mathrm{kg} /$ peso e sulfato de atropina $0,01 \mathrm{mg} / \mathrm{kg} / \mathrm{peso}$, sendo o animal extubado e, com a normalização dos sinais vitais, encaminhado à sala de pós-operatório.

TABELA 1

SEXO, PESO, IDADE, MARCHA INICIAL, PULSO INICIAL Ȧ PALPACĀO E POR AUSCULTA POR ULTRA-SONNAR DOPPLER DOS ANIMAIS DE EXPERIMENTAÇÃO

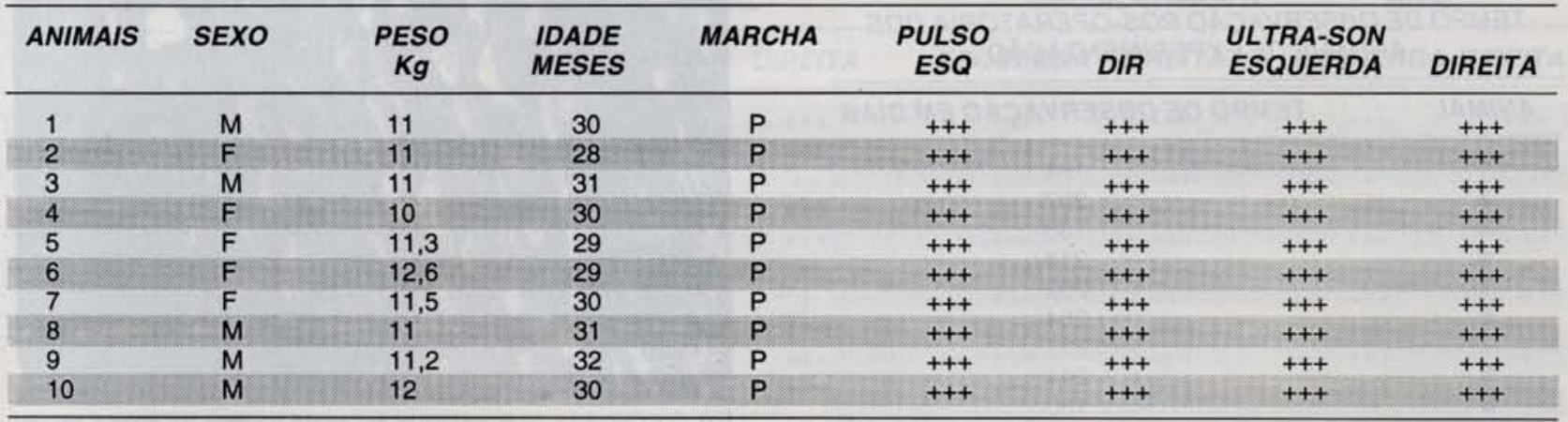

$\mathrm{F}=$ femea, $\mathrm{M}=$ macho, $\mathrm{P}=$ presente,$+++=$ amplo 
Arruda Filho M B \& Arruda M B - Comportamento da artéria caudal bovina como enxerto biológico vascular alternativo: estudo experimental em cāes. (Nota prévia). Rev Bras Cir Cardiovasc 1996; 11 (4):299-306

\section{Observação Pós-Operatória}

Os animais permaneceram isolados nos primeiros sete dias de pós-operatório, sendo observadas diariamente as funções motoras dos membros inferiores, mensurados pulso e pressão arterial nas patas esquerda e direita. Após este período, os cães foram observados a cada dez dias até o primeiro mês e a cada trinta até a reoperação para retirada do enxerto. O período de observação pós-operatória variou de 180 a 297 dias $(X=222,6)$ (Tabela 2).

Passado o período de observação pós-operatória, superior a seis meses, os cães foram reconduzidos para retirada do seguimento implantado de $\mathrm{ACB}$, utilizando-se a mesma técnica descrita para o implante.

Isoladas as artérias femorais, foram analisados os aspectos macroscópicos dos enxertos, pela sua consciência, presença de inflamação, dilatação, trombose e pulso distal ao enxerto.

As medidas da pressão arterial média foram através de um sistema composto por um cachimbo de vidro com coluna de mercúrio, conectado a um extensor e Jelco número 20, que foi introduzido antes e após o enxerto.

Após as medidas de pressão arterial média, a artéria femoral do cão foi seccionada distalmente ao enxerto, sendo avaliado o fluxo sangüíneo através do mesmo. Só então, o enxerto foi retirado, trazendo consigo um seguimento da artéria femoral do cão à montante e à jusante, medindo cerca de $1 \mathrm{~cm}$, sendo cortado longitudinalmente em duas partes, para estudo em microscopia óptica e de varredura.

A mesma técnica foi repetida no lado contralateral.

TABELA 2

TEMPO DE OBSERVACCÃO PÓS-OPERATÓRIA DOS ANIMAIS DE EXPERIMENTAÇĀO

\begin{tabular}{cc}
\hline ANIMAL & TEMPO DE OBSERVAÇÃO EM DIAS \\
\hline 1 & 184 \\
2 & 207 \\
3 & 183 \\
4 & 214 \\
5 & 252 \\
6 & 264 \\
7 & 297 \\
8 & 244 \\
9 & 180 \\
10 & 205 \\
\hline
\end{tabular}

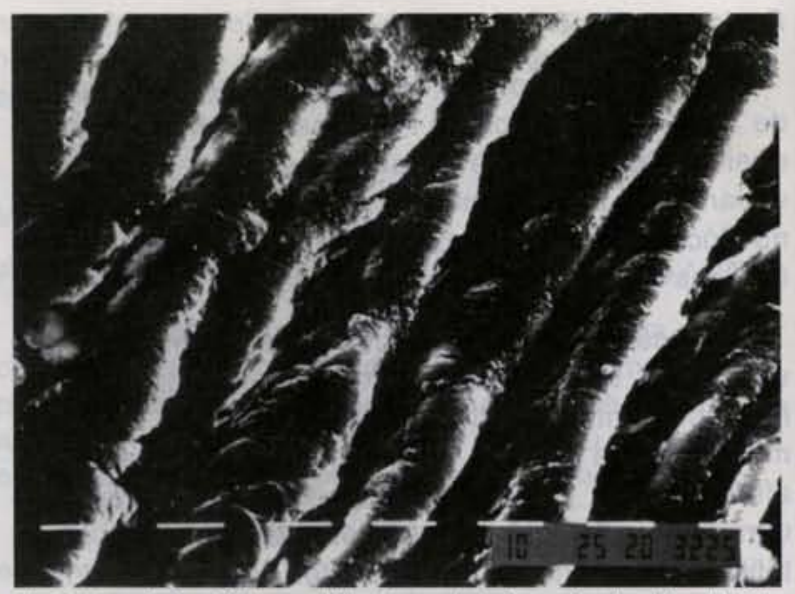

Fig.2-Enxerto da artéria caudal bovina; após tratamentos de glutaraldeído.

\section{Análise Estatística}

Os resultados foram expressos por suas médias e desvio padrão. Para análise de diferenças entre médias, aplicou-se o teste $\mathrm{T}$ de Student para amostras relacionadas. Aceitou-se como limite de rejeição da hipótese de nulidade $p>0,05$.

\section{RESULTADOS}

Não houve óbitos durante este estudo. Os cães não apresentaram alteração da marcha, não desenvolveram infecção nas incisões cirúrgicas. Os enxertos foram analisados macroscopicamente na retirada e foram observadas alteraçōes. Quanto à consistência, $3(15 \%)$ enxertos apresentaram con-

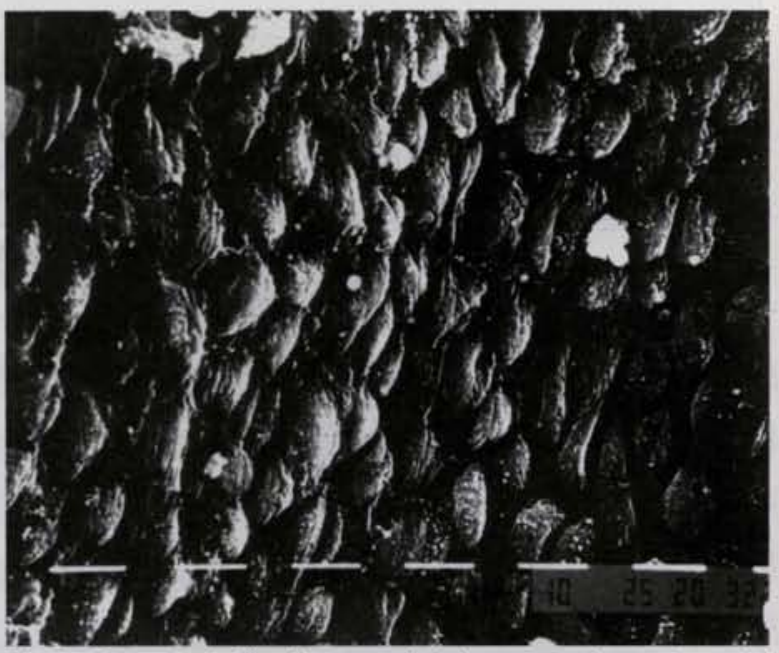

Fig. 3 - Camada de fibroblastos cobrindo enxerto da arteria caudal bovina. 
Arruda Filho M B \& Arruda M B - Comportamento da artéria caudal bovina como enxerto biológico vascular alternativo: estudo experimental em căes. (Nota prévia). Rev Bras Cir Cardiovasc 1996; 11 (4):299-306 .

TABELA 3

CONSISTÊNCIA, COLORAÇĀO, INFLAMAÇĀO, DILATAÇÃO E TROMBOSE NA RETIDA DOS ENXERTOS DE ACB

\begin{tabular}{|c|c|c|c|c|c|}
\hline ENXERTO & CONSISTÊNCIA & COLORAÇĀO & INFLAMAÇĀO & DILATAÇĀO & TROMBOSE \\
\hline 1 & A & - & $\mathrm{N}$ & $n$ & CF \\
\hline 2 & A & - & $\mathrm{N}$ & $>2 \mathrm{~mm}$ & CF \\
\hline 3 & A & - & $\mathrm{N}$ & $n$ & CF \\
\hline 4 & A & - & $\mathrm{N}$ & $\mathrm{n}$ & $\mathrm{CF}$ \\
\hline 5 & A & - & $\mathrm{N}$ & $n$ & CF \\
\hline 6 & C & - & $\mathrm{N}$ & $n$ & SF \\
\hline 7 & A & - & $\mathrm{N}$ & $n$ & $\mathrm{CF}$ \\
\hline 8 & A & - & $\mathrm{N}$ & $\mathrm{n}$ & CF \\
\hline 9 & A & - & $\mathrm{N}$ & $\mathrm{n}$ & CF \\
\hline 10 & A & - & $\mathrm{N}$ & $>2 \mathrm{~mm}$ & SF \\
\hline 11 & C & - & $\mathrm{N}$ & $>2 \mathrm{~mm}$ & SF \\
\hline 12 & B & - & $\mathrm{N}$ & $\mathrm{n}$ & $\mathrm{CF}$ \\
\hline 13 & $\bar{A}$ & - & $\mathrm{N}$ & n & CF \\
\hline 14 & A & - & $\mathrm{N}$ & $\mathrm{n}$ & SF \\
\hline 15 & B & - & $\mathrm{N}$ & $\mathrm{n}$ & CF \\
\hline 16 & B & - & $\mathrm{N}$ & $n$ & CF \\
\hline 17 & A & - & $\mathrm{N}$ & n & CF \\
\hline 18 & A & - & $\mathrm{N}$ & $\mathrm{n}$ & CF \\
\hline 19 & A & - & $\mathrm{N}$ & n & CF \\
\hline 20 & A & - & $\mathrm{N}$ & $n$ & CF \\
\hline
\end{tabular}

$\mathrm{A}=$ consistência normal, $\mathrm{B}=$ consistência endurecida, $\mathrm{C}=$ calcificaçăo focal, $\mathrm{N}=$ ausência de infecção, $\mathrm{n}=$ normal, $\mathrm{CF}=\mathrm{com}$ fluxo, $\mathrm{SF}=$ sem fluxo

sistência endurecida e $2(10 \%)$ enxertos apresentaram calcificação focal. Não houve alterações na coloração dos enxertos ou presença de inflamação. Três (15\%) enxertos, apresentavam-se trombosados. Três enxertos apresentaram aumento no diâmetro de $2 \mathrm{~mm}$ (Tabela 3 ).

Quanto ao pulso, 4 (20\%) enxertos apresentaram diminuição do pulso de amplo para fraco e 1 (15\%) enxerto apresentou diminuição de amplo para moderado, pela palpação e ausculta pelo ultra-sônar (Tabela 4).
Houve alteração da pressão arterial sistólica em $2(10 \%)$ enxertos e diastólica em $3(15 \%)$ enxertos e alteração da pressão arterial média pré e pós-enxerto em 5 (25\%) enxertos (Tabela 5).

À microscopia óptica, na camada íntima foram observados $6(30 \%)$ enxertos com trombose mural focal, e $2(10 \%)$ enxertos com trombose organizada. Não houve alterações nas lâminas limitantes externa e interna. Um (5\%) enxerto mostrou calcificação focal nas moléculas e, na adventícia, $8(40 \%)$ enxertos apresentaram congestão, $2(10 \%)$ enxertos apresen-

TABELA 4

MEDIDAS DO PULSO A PALPAÇĀO E A AUSCULTA ATRAVÉS DO ULTRA-SÔNAR

\begin{tabular}{|c|c|c|c|c|c|c|c|c|}
\hline \multirow[t]{2}{*}{$A N I M A L$} & \multicolumn{2}{|c|}{$\begin{array}{l}\text { PULSO } \\
\text { INICIAL }\end{array}$} & \multicolumn{2}{|c|}{$\begin{array}{c}\text { PALPAÇÃO } \\
\text { FINAL }\end{array}$} & \multicolumn{4}{|c|}{ ULTRA-SÔNAR } \\
\hline & ESQUERDA & DIREITA & ESQUERDA & DIREITA & ESQUERDA & DIREITA & ESQUERDA & DIREITA \\
\hline 1 & +++ & +++ & +++ & +++ & +++ & +++ & +++ & +++ \\
\hline 2 & +++ & +++ & +++ & +++ & +++ & +++ & +++ & +++ \\
\hline 3 & +++ & +++ & + & ++ & +++ & +++ & + & ++ \\
\hline 5 & +++ & +++ & +++ & +++ & +++ & +++ & +++ & +++ \\
\hline 6 & +++ & +++ & + & + & +++ & +++ & + & + \\
\hline 7 & +++ & +++ & +++ & + & +++ & +++ & +++ & + \\
\hline 8 & +++ & +++ & +++ & +++ & +++ & +++ & +++ & +++ \\
\hline 9 & +++ & +++ & +++ & +++ & +++ & +++ & +++ & +++ \\
\hline 10 & +++ & +++ & +++ & +++ & +++ & +++ & +++ & +++ \\
\hline
\end{tabular}

$+++=$ amplo,$++=$ moderado,$+=$ fraco 
Arruda Filho M B \& Arruda M B - Comportamento da artéria caudal bovina como enxerto biológico vascular alternativo: estudo experimental em cães. (Nota prévia). Rev Bras Cir Cardiovasc 1996; 11 (4):299-306 .

TABELA 5

PRESSÃO ARTERIAL SISTÓLICA, DIASTÓLICA E MÉDIA INICIAL E NA RETIRADA DO ENXERTO E FLUXO SANGÜINEO ATRAVÉS DO ENXERTO

\begin{tabular}{|c|c|c|c|c|c|c|c|c|c|}
\hline ANIMAL & $\begin{array}{l}\text { PRESSÃO } \\
\text { INICIAL }\end{array}$ & $\begin{array}{l}\text { ARTERIAL } \\
\text { RETIRADA }\end{array}$ & $\begin{array}{l}\text { ESQUER } \\
P R E-E N X\end{array}$ & $\begin{array}{l}\text { PRESSÃO } \\
\text { PÓS=ENX }\end{array}$ & $\begin{array}{l}\text { ARTERIAL } \\
\text { DIREITA } \\
\text { PRE-ENX }\end{array}$ & $\begin{array}{l}\text { MÉDIA } \\
\text { PÓS-ENX }\end{array}$ & & $\begin{array}{l}\text { FLUXO DE } \\
\text { ATRAVÉS } \\
\text { ESQUER }\end{array}$ & $\begin{array}{l}\text { SANGUE } \\
\text { ENXERTO } \\
\text { DIREITO }\end{array}$ \\
\hline 1 & $140 \times 80$ & $130 \times 80$ & 115 & 115 & 115 & 115 & & +++ & +++ \\
\hline 2 & $140 \times 90$ & $140 \times 80$ & 120 & 120 & 120 & 120 & & +++ & +++ \\
\hline 3 & $130 \times 90$ & $80 \times 50$ & 105 & 60 & 105 & 105. & & + & +++ \\
\hline 4 & $140 \times 100$ & $140 \times 100$ & 120 & 120 & 120 & 120 & * & +++ & +++ \\
\hline 6 & $140 \times 100$ & $70 \times 30$ & 120 & 50 & 120 & 50 & & - & - \\
\hline 7 & $140 \times 90$ & $60 \times 50$ & 115 & 60 & 115 & 55 & & +++ & - \\
\hline 8 & $135 \times 110$ & $130 \times 100$ & 125 & 125 & 125 & 125 & & +++ & +++ \\
\hline 9 & $140 \times 60$ & $135 \times 80$ & 115 & 115 & 115 & 115 & & +++ & +++ \\
\hline 10 & $130 \times 90$ & $130 \times 80$ & 115 & 115 & 115 & 115 & & ++ & ++ \\
\hline
\end{tabular}

$+++=$ amplo, $++=$ moderado,$+=$ fraco

taram infiltrado inflamatório e $9(45 \%)$ apresentaram infiltrado inflamatório com neoformação vascular.

A microscópia de varredura mostrou reepitelização total em $12(60 \%)$ enxertos, ilhas de reepitelização em $3(15 \%)$ enxertos e presença de células inflamatórias em $6(30 \%)$ enxertos. Houve depósito de fibrina em 5 (25\%) enxertos e trombose total em $3(15 \%)$ enxertos .

\section{COMENTÁRIOS}

A busca por um substituto vascular alternativo, principalmente para pequenas e médias artérias, tem proporcionado ensaios os mais diversos ao longo do tempo.

A utilização clínica dos heteroenxertos arteriais bovinos surgiu da necessidade de construção de fístulas arteriovenosas em pacientes portadores de insuficiência renal crônica e de doença vascular periférica, onde os vasos não eram adequados para tal fim.

Entre os heteroenxertos arteriais bovinos, os de artéria carótida são os mais utilizados, possuem parede espessa, textura e diâmetro aumentados para operação de pequenas artérias $(22,23)$. Em seguida, surgiram no mercado as artérias torácicas internas bovinas, que, apesar da textura adequada da parede, possuem diâmetro aumentado para as operações de pequenas artérias (18).

A escolha da artéria caudal bovina como enxerto vascular alternativo deve-se ao seu comprimento médio de $70 \mathrm{~cm}$, suficiente para atingir ou substituir segmentos de artérias distais, possuir expessura da parede apropriada, diâmetro compatível variando de 2 a $4 \mathrm{~mm}$ e ser de fácil obtenção.
Os métodos de preservação e conservação dos heteroenxertos, embora não tenham alcançado os objetivos desejados, sāo satisfatórios e o glutaraldeído e o formaldeído foram utilizados.

O período de observação pós-operatória variou de seis a dez meses, portanto superior à maioria dos estudos experimentais neste campo, que, em média, são inferiores a seis meses de observação clínica (23-27).

As alteraçōes de consistência nos trabalhos experimentais utilizando-se heteroenxertos arteriais bovinos mostram variações nos resultados de $10 \%$ a $50 \%$, apresentando desde pequenos depósitos de cálcio até metaplasia com formação de tecido osteióide e medula óssea, como demonstrado por ROSEMBERG et al. (22) Macroscopicamente, no presente estudo, foram encontrados consistência endurecido em 3 enxertos e 2 apresentaram calcificação focal. Estes achados foram confirmados histologicamente como calcificação focal nos 5 casos.

Os animais não apresentaram inflamação da ferida operatória ou alteração da coloração dos enxertos.

A incidência de trombose total nos heteroenxertos arteriais bovinos em estudos realizados por SOMOGY et al. (23), De TAKATS et al. ${ }^{(28)}$ e VAS. CONCELOS ${ }^{(27)}$ em aorta abdominal de cães, são variáveis e possuem média em torno de $25 \%$. Em estudos de DALE \& LEWIS (24), em cirurgia arterial restauradora fêmoro-poplítea apresentam incidência em torno de $40 \%$ de trombose total. Este estudo realizado em artéria femoral de cães, tecnicamente mais difícil que em aorta abdominal, mostrou $3(15 \%)$ enxertos, com trombose total em até dez meses de seguimento pós-operatório. 
Arruda Filho M B \& Arruda M B - Comportamento da artéria caudal bovina como enxerto biológico vascular alternativo: estudo experimental em cães. (Nota prévia). Rev Bras Cir Cardiovasc 1996; 11 (4):299-306 .

De TAKATS et al (28), ROSEMBERG et al. (22) e NAKATA et al. (25) demonstraram que a dilatação dos enxertos arteriais bovinos em estudos experimentais, está relacionada com o tipo de preservação realizada. $\mathrm{O}$ aumento de dois milimetros em 3 $(15 \%)$ enxertos, ocorridos neste estudo é menor que os resultados apresentados na literatura.

Apesar de não haver referência em outros estudos na literatura, o acompanhamento do pulso à palpação e a ausculta pelo ultra-sônar e as medidas da pressão arterial sistólica, diastólica e média, em períodos pré-estabelecidos, foram usados como mais um parâmetro auxiliar importante na avaliação da perviabilidade dos enxertos. Houve alteração do pulso de amplo para fraco em $4(20 \%)$ enxertos e diminuição de amplo para moderado em $1(5 \%)$ enxerto. Houve alteração da pressão arterial sistólica em $2(10 \%)$ animais, diminuição da pressão arterial diastólica em 1(5\%) animal e diminuição da pressão arterial média pré e pós enxerto em 5 (25\%).

PARÉ et al. (26) relataram como achados histopatológicos mais freqüentemente encontrados quando da retirada dos enxertos, em estudos experimentais utilizando heteroenxertos bovinos na sequência: trombose focal, principalmente nas linhas de sutura, que são substiuídos por tecido de cicatrização e recobertos com fibroblastos, com aspecto endotelial. Outro achado comum é hiperplasia intimal, principalmente nas linhas de anastomose, infiltrado linfoplasmocitario e calcificação focal na adventícia. Neste estudo, encontrou-se infiltrado linfoplasmocitario focal em $100 \%$ dos enxertos e calcificação focal em $25 \%$ deles. A presença de trombose focal inicial em $6(30 \%)$ enxertos em período superior a seis meses não coincide com os resultados iniciais apresentados na literatura. O recobrimento do enxerto por material cicatricial (fibrina) e a presença da camada de fibroblastos em toda a extensão do enxerto, em $60 \%$ e parcial em $30 \%$, seguem os achados da literatura; assim como o aparecimento de células inflamatórias esparsas e plaquetas, quando da rotura da lâmina interna ao nível da sutura distal em 1 caso $(5 \%)$, como relatado por SOMOGY et al. (23). Não foram encontrados depósitos de gordura nas paredes dos enxertos, como relatados por ROSEMBERG et al. (22) e HARJULA et al. (29). Houve invasão de capilares através do enxerto em 2 casos $(10 \%)$ na sutura proximal dos enxertos, como descrito por SOMOGY et al. (23).

No presente trabalho não foi encontrada reação de rejeição importante ao enxerto, provando eficácia na redução da antigenicidade, apesar da reação linfoplasmocitária discreta na adventícia. Foram demonstrados outros achados importantes, como ausência de infecção e re-epitelização total em $60 \%$ dos casos e parcial em $30 \%$, em período superior a seis meses.

\section{CONCLUSÕES}

A ausência de infecção e rejeição e rápida endotelização, demonstram a receptividade da utilização da artéria caudal bovina como enxerto vascular alternativo.

O método de preservação utilizado reduziu a antigenicidade, fixou as proteínas, mantendo a estabilidade do enxerto.

Arruda Filho M B \& Arruda M B - Bovine tail artery as an alternative vascular graft: experimental study in dogs. (Preliminary report). Rev Bras Cir Cardiovasc 1996; 11 (4): 299-306.

ABSTRACT: Twenty segments of bovine tail artery treated with glutaraldehyde were interposed into 10 dog's femoral arteries. The post-operative period varied from 183 to 297 days. Motor function, pulses, systolic, diastolic and median blood pressure and blood flow throught the graft, as well as consistence, dilatation and color in the follow-up period were analysed. There were no deaths, motor function or inflammatory changes. There were $15 \%$ of total thrombosis, focal wall thrombosis $(30 \%)$, advential focal linfoplasmocitory reaction $(100 \%)$, myocells calcification (15\%), total reendotelization $(60 \%)$ and partial $(30 \%)$. Our data show that bovine tail artery has a diameter adequacy with small arteries, is long enough to replace or reach several distal arterial segments, has easy epitelization, and absence of cytological reation, allowding its use as an alternative graft.

DESCRIPTORS: Femoral artery, surgery. Bioprosthesis, methods. Biocompatible materials. 
Arruda Filho M B \& Arruda M B - Comportamento da artéria caudal bovina como enxerto biológico vascular alternativo: estudo experimental em cães. (Nota prévia). Rev Bras Cir Cardiovasc 1996; 11 (4):299-306 .

\section{REFERÊNCIAS BIBLIOGRÁFICAS}

1 Glieny Jr. K S, Flege Jr. J B, Callara G M, Tood J C - Twenty years follow up of saphenous vein aortocoronary bypass. Ann Thorac Surg 1992; 53: 258-62.

2 Lowrie G M, Morris Jr. G C, Earle E - Long term result of coronary bypass surgery: analysis of 1698 patients follow 15 to 20 years. Ann Surg 1991; 213: 377-85.

3 Kalan J M \& Roberts W C - Morphologic fidings in saphenous veis used as coronary artery bypass conduits for longer than one year. Necropsy analysis of 53 patients, 123 saphenous veins and 1565 five milimiter segments of vein. Am Heart J 1990; 119: 1164-84.

4 Cox J L, Chiasson D A, Gotlieba I - Strange in a strange land. The pathogenesis of saphenous vein graft stenosis with emphasis on strutural and functional differences between veis and artery. ProgrCardiovasc Dis 1991; 34: 45-68.

5 Lytle B W, Loop F D, Cosgrove D M, Ratclif N B, Easley K, Taylor P C - Long term 15 to 20 years) serial studies of IMA and saphenous vein coronary bypass grafts. J Thorac Cardiovasc Surg 1985; 89: 248-59.

6 Lytle B W, Loop F D, Taylor P C - Vein graft desease. The clinical impact of stenoses in saphenous vein grafts to coronary arteries. J Thorac Cardiovasc Surg 1992; 103: 831-40.

7 Loop F D - Use of in situ and free internal thoracic artery for miocardial revascularization. J Cardiovasc Surg 1986; 1: 205-16.

8 Loop F D, Lytle B W, Cosgrove D M, Goldinglair R, Taylor P C, Stwart R W - Free aorta-coronary IMA grafts: late results. J Thorac Cardiovasc Surg 1986; 92: 827-31.

9 Zeff $\mathrm{R} \mathrm{H}$, Kongtahworn $\mathrm{C}$, Jamrone $\mathrm{L} A$ - IMA versus saphenous vein graft to the left anterior descending coronary artery: prospective randomized study with ten year follow up. Ann Thorac Surg 1988; 45: 533-6.

10 Buche M, Schoevaerdts J C, Lovagre Y - Use of inferior epigastric artery for coronary bypass. J Thorac Cardiovasc Surg 1992; 103: 665-70.

11 Chui C - Why do radial artery grafts for aortocoronary bypass failure? A reapraisel. Ann Thorac Surg 1993; 22: $520-3$.

12 Mueller D K, Blakeman B D, Pickleman J-Free splenic artery in aorto coronary bypass. Ann Thorac Surg 1993; 55: 162-3.

13 O'Neil G S, Chester H H, Allen S P - Endothelial function of human gastroepiploic artery. Implications for its use as a bypass graft. $J$ Thorac Cardiovasc Surg 1996; 102: 561-5.

14 Suma H, Wambichi $Y$, Futura S Takeuchi - Does use of gastroeploic artery graft increase surgical risk? J Thorac Cardiovasc Surg 1993; 101:121-5.
Chara R B, Johnson D C, Nunn G R, Cartmill T B Aortocoronary bypass grafiting with polytetrafluoretlylene conduits: early and late outcome in eight patients. J Thorac Cardiovasc Surg 1987; 94: $132-4$.

16 Sapsford R N, Oakley G D, Talbot S - Early and late patency of expanded polytetrafluorethylene vascular grafts in aorto coronary bypass. J Thorac Cardiovasc Surg 1981; 81: 860-4.

17 Tordoir J N, Van-der-plos J P, Jacobs M I, Kitslaar P I - Factors determining outcome of crural and pedal revascularization for critical limb ischaemia. Eur $J$ Vasc Surg 1993; 7: 82-6.

18 Donzeau G P, Jovatt G, Vouhe P R et al. - Coronary bypass with bovine internal mammary graft. Arch Mal Coeur 1990; 83: 1811-5.

19 Foran R F, Shore E H, Levin P M, Treiman R L - Bovine heterografts for hemodialysis. Wester J Med 1975; 123: 268-74.

20 Harder $F$ \& Landmann $J$ - Trends in access surgery for hemodialysis. Surg Ann 1984; 16: 135-49.

21 Rosemberg N, Lora G H, Henderson J, Bothwell J W, Goughran F R L - Collagen arterial graft of bovine origen: seven years observations in dogs. Surgery 1970; 67: 951-6.

22 Rosemberg N, Henderson J, Lord G H, Bothwell J W, Gaughran E R L . The use of enzymetreated heterografts as segmental arterial substitutes: follow up observations of five-year-old implants. Arch Surg $1961 ; 83: 950-5$.

23 Somogy E, Sotony P, Nemes A, Soltesz E - experimental observation regarding the applicability of calf carotid xenograft in vascular surgery. $J$ Thorac Cardiovasc Surg 1980; 21: 723-6.

24 Dale W A \& Lewis M R - Modified bovine heterografts for arterial replacement. Ann Surg 1969; 169: 927-46.

25 Nakata Y, Hayakawa N, Nakagami K, Ikezawa T, Maeda $S$ - Late changes in bovine graft. Case report. Jpn J Surg 1982; 12: 368-71.

26 Paré G, Guido I N R, Marois M - Evaluation de deux heterograffes bovines commerciales pour le replacement vasculaire. J Chir (Paris) 1980; 117: 331-9.

27 Vasconcelos F J O M - Utilização da artéria mamária bovina tratada pelo glutaraldeido em aorta abdominal de cão: análise morfologica de 11 enxertos após 180 dias de implante. [Tese. Mestrado] Recife: Faculdade de Medicina da Universidade Federal de Pernambuco, 1988. 152p.

28 De Takats G, Thompson J D, Dolowy W C - Bovine arterial grafts: an experimental study. Ann Surg 1959; 150: 1017-24.

29 Harjula A, Nickels J, Matilla A S - Histological study of glutaraldehyde processed vascular graft of biological origin. Ann Chir Gynecol 1980; 69: 252-62. 